\title{
FREACSIM - A Framework for Creating and Simulating Real-Time Capable Network on Chip Systems and Applications
}

\author{
Dominik Schoenwetter \\ Chair of Computer Science 3 \\ (Computer Architecture) \\ Friedrich-Alexander-University \\ Erlangen-Nürnberg (FAU) \\ Martensstr. 3, 91058 \\ Erlangen, Germany \\ dominik.schoenwetter \\ @fau.de
}

\author{
Ronald Veldema \\ Chair of Computer Science 2 \\ (Programming Systems) \\ Friedrich-Alexander-University \\ Erlangen-Nürnberg (FAU) \\ Martensstr. 3, 91058 \\ Erlangen, Germany \\ ronald.veldema@fau.de
}

\author{
Dietmar Fey \\ Chair of Computer Science 3 \\ (Computer Architecture) \\ Friedrich-Alexander-University \\ Erlangen-Nürnberg (FAU) \\ Martensstr. 3, 91058 \\ Erlangen, Germany \\ dietmar.fey@fau.de
}

\begin{abstract}
This paper presents the new Framework for Real-time capable Embedded system and ArChitecture SIMulation (FREACSIM), a highly configurable full-system simulation environment enabling and easing the modeling, simulation and verification of Network on Chip architectures for hard real-time systems. The framework is mostly geared towards software developers, supporting them in the simulation of NoCs at an instruction accurate level and offers a broad variety of real-world hardware components as part of the integrated virtualization toolbox.

FREACSIM provides a software-based routing strategy between nodes, with a single node consisting of a processor and required peripherals for enabling real-time capability. This allows a flexible and independent comparison of currently implemented hardware strategies, as well as an easy adaption to better suit new hardware needs. The softwarebased routing, as well as distributed applications that can be implemented for the NoC hardware design, are able to use the real-time operating system eCos, which is part of our framework. As a result, real-time capable software can be implemented for, and tested on, complex NoC systems.

We demonstrate the flexibility and the benefits of our framework with a set of applications (use cases), which cover typical heavy and light load distributions between communication and computation.
\end{abstract}

\section{Categories and Subject Descriptors}

C.3 [Computer Systems Organization]: Special-Purpose and Application-Based Systems-Real-time and embedded systems; D.4.7 [Operating Systems]: Organization and Design-Real-time systems and embedded systems; I.6 [Si- mulation and Modeling]: Miscellaneous

\section{General Terms}

Design, Performance

\section{Keywords}

Embedded, Network on Chip, Real-Time, Simulation, Software-Based Routing

\section{INTRODUCTION}

Over the last few years, parallel computing has gained more and more attention in different sectors of embedded industry. Most importantly in the automotive domain where hard real-time requirements and many other life critical constraints exist. Although, much innovation in this area is driven by entertainment systems and visualization, the amount of required compute performance also increases in more sensible areas, such as engine controllers and ambient sensor data acquisition and processing, e.g. LIDAR (LIght Detection And Ranging). For a long time, single core designs were powerful enough to satisfy performance requirements. As we slowly reached similar constraining factors as in desktop environments almost a decade ago, these demands cannot be satisfied any longer. As a consequence, even real-time requirements could not be met any longer. Thus, the change to multi-core designs was a necessary step to increase performance and guarantee those hard real-time requirements. At the moment, more and more functionality is added to real-time capable applications and, as a consequence, more computing power is needed to satisfy the requirements. That resulted in Network on Chip architectures and systems, respectively, where hardware developers dissociate from traditional bus systems. Even if these systems are not used as the standard in current electronic systems, they will play a central role in the future of the embedded domains where hard real-time requirements exist.

From our point of view, the domains, where hard realtime is required, have many ideas how the respective NoChardware architecture can look like for their use cases, but often have none concrete idea what is the best. As a consequence, the likelihood of changes to the hardware layout 
during the design phase is very high. To avoid that often difficult and cost-intensive effort, the usage of an environment that emulates the embedded Network on Chip hardware would be of great advantage. By using this methodology the hardware can be modified, whenever necessary, without the effort of actual hardware redesigns. That is a big advantage for software developers as well. During the period of time of the redesign, software developers have no actual hardware to implement software for. This can result either in stagnation or bad code that does not exploit the features of the redesigned hardware. As a result, software developers often want a full-system simulation environment that enables them to develop and test their software quickly on emulated hardware, does not require too much time for the simulation and enables them flexibility in many ways. Concerning to fast simulation times and software evaluation, the instruction accurate simulation level is very well suited. It's not as detailed as the cycle accurate level or levels below, but full-system simulation is possible, not only at a functional level. In comparison to the instruction accurate level, the cycle accurate level is very slow concerning to simulation speed. Weaver and McKee showed that there can be discrepancies of hours up to days [23].

A software developer also wants flexibility concerning to routing and communication. Routing algorithms, like XY routing [16] or wormhole routing [20], that are implemented in hardware, do not offer flexibility from a point of view of switching and routing. Often some leeway is required in the scope of flexibility, independence and performance. A software-based routing strategy enables the just mentioned flexibility. As a consequence, an easy adaption to better suit new hardware needs is possible.

On these grounds, we developed the Framework for Realtime capable Embedded system and ArChitecture SIMulation (FREACSIM), that targets software developers in the first instance. FREACSIM is an instruction accurate fullsystem simulation environment that enables the creation and simulation of a large number of real-time capable embedded NoC architectures in a fast way. Because the framework is a full-system environment, software developers have the possibility to simulate their applications on real-time capable embedded NoC architectures. Furthermore, FREACSIM provides a software-based routing solution that enables the implementation of distributed and real-time capable applications. The possibility of implementing real-time capable applications is obtained by the real-time operating system eCos (embedded Configurable operating system, [4]), which is included into our software-based routing and elucidated in section 4 .

To avoid starting from scratch, we decided to use an existing instruction accurate simulation environment as the simulation engine of our framework which can be adapted to our needs. This allows more flexibility in our designs, because emulated hardware components are already available. We choose Open Virtual Platforms provided by Imperas. With the aid of OVP it is possible to build single- up to many-core hardware architectures, add desired peripherals and simulate real application code [14]. Because of the ability to establish multi- and many-core architectures running real application code, it is possible to develop distributed applications that can be simulated, verified and evaluated. That is an important feature for our work and one of the reasons why we chose OVP as the virtual environment. An- other reason why this environment was chosen is, that OVP offers a wide range of processor and peripheral models for the simulation. As a consequence FREACSIM can be extended to more hardware components, if necessary. Because OVP is an instruction accurate simulator, as explained in section 3 , the simulations are very fast.

This paper is organized as follows. The next section shows an overview of existing simulation environments and solutions, as well as further related work. In section 3 and section 4, a short overview of OVP and eCos is given. Section 5 describes the framework FREACSIM and its tools. The software applications (use cases) we implemented for demonstrating the flexibility and the benefits of our framework are illustrated in section 6 . Afterwards, the results of our measurements are shown (section 7). The paper concludes with a short summary and an outlook on future work.

\section{RELATED WORK}

There is a wide range of free as well as commercial Network on Chip simulators and frameworks available. One commercial variant is NetSim [11]. NetSim is provided by Boson and uses Boson's proprietary simulation and routing tools. This simulator is only available for Windows and the focus is on routing and switching. NetSim enables the simulation of routers, switches as well as PCs. Supported are 42 different routers and 6 different switches. The focus of this simulator is not on embedded Network on Chip systems.

One free variant of a network simulation tool is Graphite, presented by Miller et al. in 2010 [19]. This simulator offers the possibility to simulate hundreds or even thousands of cores. Graphite is not a complete cycle-accurate simulator, it uses different techniques to provide accurate performance results. The simulation environment offers processors, a memory subsystem, cache models as well as a network for realizing interconnections. All these models use further analytical timing models to guarantee accurate results. However, the focus of Graphite is not on embedded systems.

The probably most widespread free and open source emulation environment is QEMU [8]. In most cases, QEMU is used to run one operating system on another, e.g. Windows on Linux. Because QEMU can be stopped during execution and the current state can be examined, debugging is also a use case. QEMU supports a couple of embedded processors, but does not target the embedded domain directly.

A simulation environment that focuses on the network simulation of NoC systems is BookSim [15]. This simulator is designed to be cycle accurate, but no full-system simulator that is able to simulate nodes and processors of nodes, respectively. The simulator provides accurate modeling of network components as well as flexibility. Flexibility is given by the possibility of configuring network parameters like the topology, flow control or the routing algorithm that shall be used. Furthermore, the microarchitecture of the router can be configured, including the management of buffers and different allocation schemes.

An environment that focuses on virtual prototyping of multi-processor system on chips (MP-SoC) is SoCLib [22]. SoCLib provides a wide range of processor and peripheral models, for example MIPS32 and ARM. Furthermore, the usage of real-time operating systems like eCos is supported. This environment enables simulations at the cycle accurate level as well as the bit accurate level. Because all models are 
written in SystemC [6], the ability to simulate at transaction level is given, too.

A cycle accurate Network on Chip interconnection model called Garnet [7] was published in 2009 by Agarwal et al. The model is embedded into the GEMS (General Executiondriven Multiprocessor Simulator, [5]) environment. Details, such as flit-level input buffers or routing logic are modeled. GARNET in conjunction with GEMS provides a detailed, as well as accurate, timing model of the memory system. They evaluated the benefits and the potential of their model by comparing it against the network model provided by GEMS. Their setup consisted of 16 in-order 2-way SPARC processors with $64 \mathrm{~KB} \mathrm{~L} 1 \mathrm{I} \& \mathrm{D}$ caches, L2 and direct caches, as well as 4 memory controllers and the respective NoC interconnection model. GEMS is no longer under active development. The development has been shifted to the gem5 simulation system, an open source software, which is discussed in the next paragraph.

The gem5 simulation environment [9] combines the benefits of the M5 [10] and the GEMS environments. M5 is a configurable simulation environment offering multiple ISAs (instruction set architectures) as well as various CPU models. The CPU can be configured to operate on different levels of detail and accuracy. In combination with GEMS, gem5 provides a detailed and flexible memory system as well as interconnection models. A wide range of instruction set architectures (e.g. x86, ALPHA or MIPS) is supported by gem5. This simulation environment is not designed to be pure instruction accurate and targets the embedded domain partially.

Madsen et al. published a paper on a modeling environment for embedded System-on-Chip (SoC) designer, dealing with multiprocessor architectures [17]. They are focusing on real-time applications and the interconnection of single processors using NoCs. The base for their modeling environment is SystemC. As a consequence, the environment is neither complete cycle accurate nor complete instruction accurate what impacts the simulation performance in comparison to a complete instruction accurate environment.

Recently, Schoenwetter et al. made eCos available to the simulation environment OVP [21]. They validated their work by showing that their implementation of an engine control unit software that uses eCos and was simulated within Open Virtual Platforms works. Imperas, the founder of Open Virtual Platforms, published that work on their website [1].

\section{THE SIMULATION ENVIRONMENT OPEN VIRTUAL PLATFORMS ${ }^{\mathrm{TM}}$}

We use Open Virtual Platforms (OVP) as the engine that drives the simulation of our Network on Chip architectures.

The instruction accurate simulation technology from Open Virtual Platforms was developed for high performance simulation. The technology enables debugging applications, which run on the virtual hardware, as well as analysis of virtual platforms containing multiple processor and peripheral models. The OVP simulation technology is extensible. Furthermore, it provides the ability to create new processor models and other platform components by writing $\mathrm{C}$ or $\mathrm{C}++$ code that uses application programming interfaces (APIs) and libraries supplied as part of OVP [13].

OVP multi-component platforms (multi-processor plat- forms or single core platforms with a specified number of peripherals) are not working simultaneously. For efficiency, each processor and peripheral, respectively, advances a certain number of instructions in turn. So in multi-component simulations a single component is simulated until it has signaled that it has finished its quantum. The quantum is defined as the time period in which each component in turn simulates a certain number of instructions [13]. The just mentioned and changeable time period is called a time slice. Simulated time is moved forward only at the end of a quantum. This can create simulation artifacts, for example where a processor spends time in a wait loop, while waiting for the quantum to finish. To avoid this the quantum has to be set very low (perhaps even to one, which will have a significant impact on simulation performance) so that the measurements will not be affected by this simulation artifacts. The time slice can be adjusted in the simulator settings [14]. The simulation can only figure out how many instructions were executed. Assuming a perfect pipeline, where one instruction is executed per cycle, the instruction count divided by the mips rate (millions of instructions per second) would give the amount of time the program runs. The OVP-simulator provides the possibility for measuring instruction counts within a program. As a consequence, the instruction counts for specific code snippets can be recorded.

\section{OVERVIEW ABOUT ECOS}

eCos (embedded configurable operating system) is a free real-time operating system designed for embedded systems. A wide variety of popular embedded processor architectures is supported. This makes eCos a good choice for end users that have to deal with many diverse hardware architectures. The design of eCos corresponds to a configurable component architecture consisting of several key software components such as the kernel and the HAL (Hardware Abstraction Layer). This allows the construction of a complete embedded system from these reusable software components. Furthermore, different configuration options within the software component can be chosen and unused software components can be removed. To summarize, an operating system that specifically matches the requirements of an application can be created.

An application that uses eCos runs as a part of the operating system, contrary to operating systems like Linux. Thus, an eCos application is a monolithic block where the operating system and the application are not considered separately.

eCos provides a multilevel queue scheduler and a bitmap scheduler. The multilevel queue scheduler is able to execute multiple threads of the same priority level. This scheduler allows preemption between the different priority levels. The bitmap scheduler is able to execute threads at multiple priority levels, too. However, just a single thread can exist at each priority level. As a result, the bitmap scheduler is very efficient because the same priority level for two threads is forbidden what simplifies the scheduling algorithm [18].

Our framework supports the usage of both schedulers.

\section{THE FRAMEWORK FREACSIM}

The framework FREACSIM is able to generate simulation models of various real-time capable embedded Network on Chip architectures and to simulate these simulation mod- 


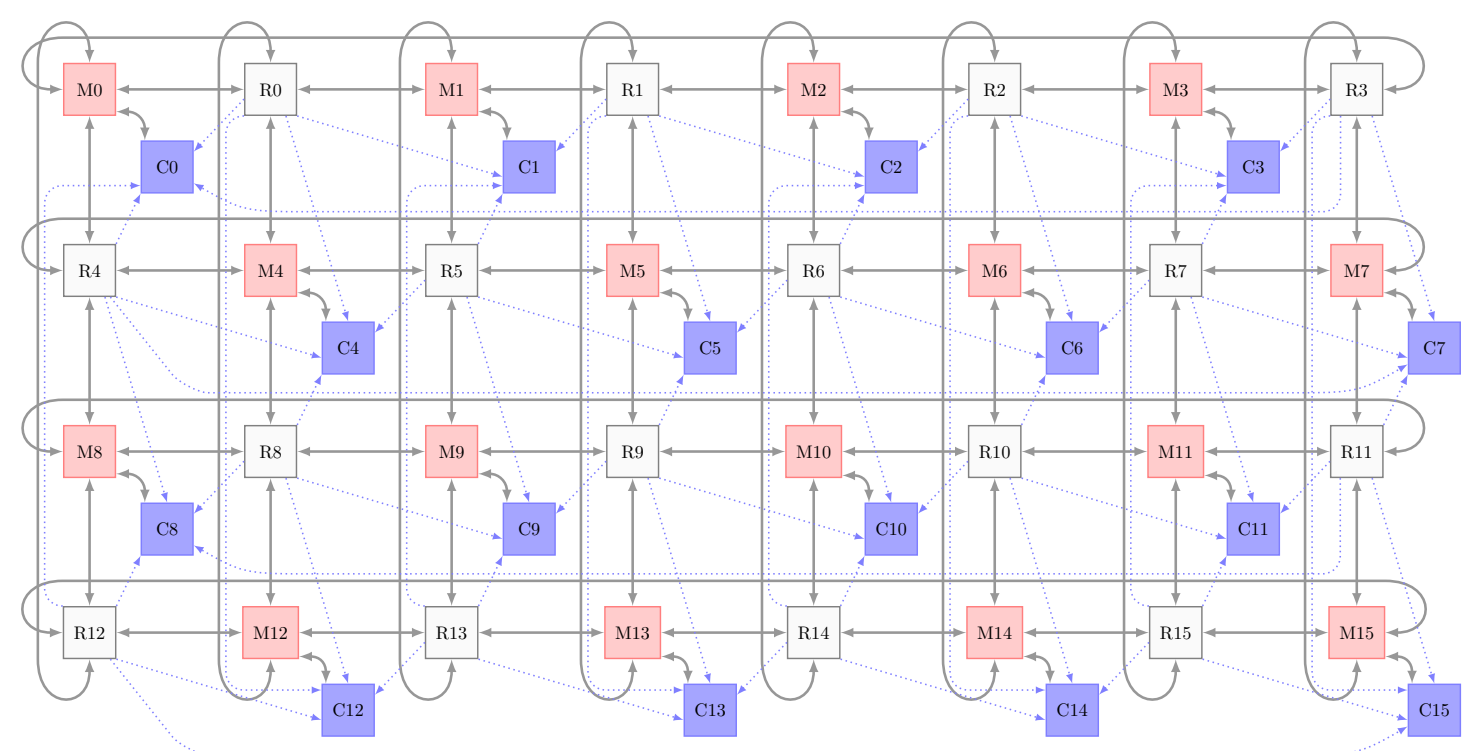

Figure 1: $4 \times 4$ torus-2D architecture with routing and computing nodes as well as notification of computation cores using interrupts if new data have arrived.

els afterwards. For each of those various hardware architectures, a real-time capable, and software-based, routing library can be generated. The real-time capability is achieved by using the real-time operating system eCos, which runs on every node core that requires real-time capability and is encapsulated in our routing library with a corresponding API. That API can be used by a software developer to implement distributed and real-time capable applications. A brief overview of eCos can be obtained from section 4 . Concerning to the hardware, FREACSIM allows the usage of different topology schemes for the interconnection of the single nodes. At the moment, the topologies star, ring, grid$2 \mathrm{D}$ and torus-2D are implemented and can be used within a design. Figure 1 shows an example and a visualization of one possible architecture $(4 \times 4$ torus- $2 \mathrm{D})$ that can be generated and simulated with FREACSIM. Figure 2 shows an overview of the framework.

Depending on the user input, like the topology or the number of nodes to use, the tool noc-generator creates a NoC-Design. The representation of this Design is within a self defined XML format, what we call an XML hardware description. This XML hardware description is the input for the tool xml-to-sim-model, which generates a complete Open Virtual Platforms simulation model out of the XML description. We decided to introduce this intermediate XML format for two reasons. First, the user has the ability to write self defined hardware descriptions in the given XML format, what results in more flexibility and independence. If there would be no XML interface, the tools noc-generator and xml-to-sim-model could be seen as one tool that is more complex. As a consequence, the user could only use the hardware descriptions that are generated by the tool nocgenerator. Second, if a further simulation environment shall be added to the framework, only the adaption of the tool xml-to-sim-model has to be done.

The XML hardware description contains required components, like processors or memories, as well as the interconnection of that single components that define the NoC-

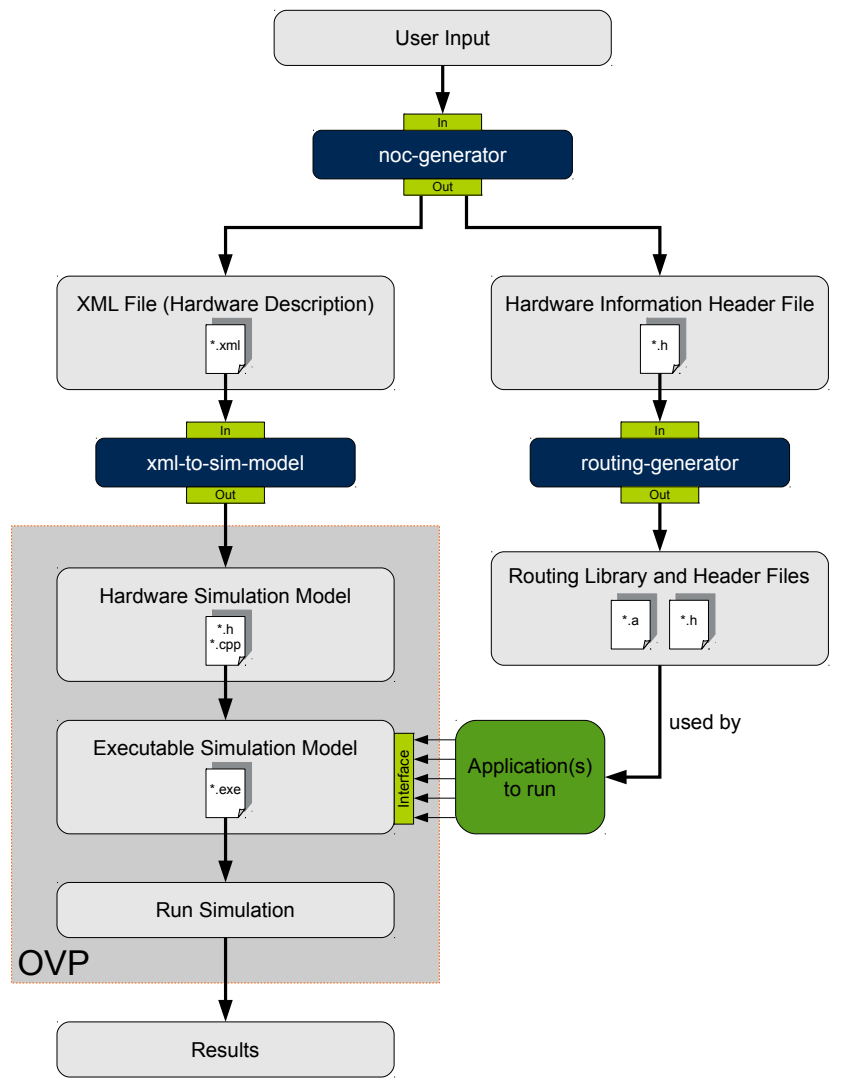

Figure 2: Overview of the tools and components of the framework FREACSIM.

Design and architecture, respectively. An example of such a NoC-Design is shown in Figure 1. Furthermore, the tool noc-generator creates a header file that contains information 
about the created hardware design, e.g. which node is interconnected directly with another node or at which address a memory (message buffer) is accessible for a processor of a node.

This hardware information header file is the input for the the tool routing-generator. That tool creates a software library and the required header files, that enables the (software-based) communication and routing between nodes in the design. The header files contain the prototypes of our API-functions, that have to be used to communicate between nodes, for example to send and receive data packets/messages. The library also encapsulates the necessary libraries of eCos, to enable the real-time capability on the nodes.

Now the user is able to implement a distributed application for the NoC-Design. As already mentioned, the applications for the single node processors have to use our APIfunctions to enable the communication between nodes. We provide a set of software applications and use cases, respectively (see section 6), that use our API.

After the implementation of the single programs of the distributed application, that single programms have to be loaded into the processor memories of the corresponding nodes, what is done using an interface provided by OVP. The user has the possibility to control the simulation using parameters. One particular parameter for the simulation is the time slice, which was elucidated in section 3 . The time slice controls the simulation speed. After the simulation has terminated, the results can be inspected and evaluated.

\subsection{Architectures of Nodes}

We distinguish the nodes in our designs (cf. Figure 1) into routing nodes and computation nodes. The computation nodes shall only perform actions of applications and shall not be busy with routing tasks. As a consequence, the routing nodes take care of the routing. It is also possible to disable the routing nodes what results in an architecture, where no distinction between routing and computation nodes is made and the routing as well as the computations have to be done by the same node. The focus, however, is on architectures that distinguish between routing and computation nodes, because node cores of (hard) real-time capable embedded systems normally don't want to spent time for routing tasks, because the computing time is required for CPU-intensive computation tasks.

The base for the implementation of our software-based routing, that uses the store and forward algorithm, are single shared memories, that are connected to some computation and routing nodes, respectively. These shared memories act as buffers, the store and forward routing algorithm works with (see Figure 1). Which computation nodes and which routing nodes are connected to a shared memory depends on the chosen topology and if routing nodes are enabled or not.

A computation node has a fixed architecture (Figure 3), a routing node has two possible architectures.

One single computation node consists of five hardware components. These five components are required because the real-time capability is achieved by using the real-time operating system eCos (see also section 4) that runs on every computation node in our NoC designs. The first component is a UART controller/interface. This interface is used for input and output calls of C-functions like printf or getc. The

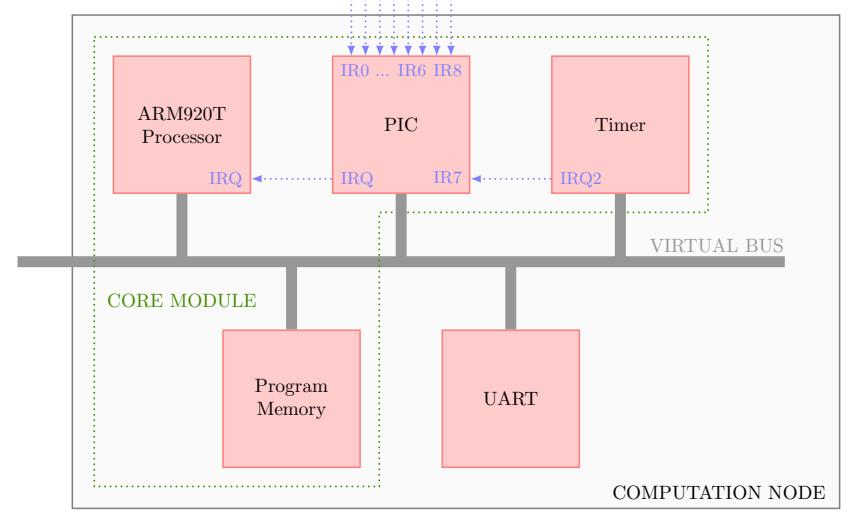

Figure 3: Architecture of a computation node.

association of the other four hardware components is called a core module. The core module consists of a processor, a programmable interrupt controller (PIC), a timer and some core local program memory. This memory is loaded with the application that uses our routing library, which contains the necessary eCos libraries. The timer generates periodically an interrupt on pin IRQ2, which is defined as the scheduler clock of eCos, and is forwarded to the processor's interrupt pin (IRQ). All components are connected to a virtual OVP bus (see [14]). That bus is the interface where a shared memory (buffer) is connected to, independent of the chosen topology (cf. Figure 1).

A routing node can consist of the same components as a computation node (same processor, etc.) and one additional component called a signal generator. The signal generator component is a hardware component that is required for triggering interrupt pins on computation node cores. If the notification for new data of a computation node shall be realized using interrupts, the pins IR 0 to IR 6 and IR 8 of the computation node interrupt controller are used to connect a corresponding pin of the signal generator component. As a consequence, it is possible to send interrupts from a routing node/core to a computation node/core for notification of new data.

Because the routing node has the same architecture as the computation node, with the exception of the signal generator component, eCos can also run on the routing node. Thus, the real-time capability is given on a routing node, too. The other possibility of the architecture of a routing node is that the real-time capability is disabled and the routing takes place without eCos. In that case, a routing node requires a processor, some processor local memory and the just mentioned signal generator component for triggering interrupts, if desired. At the moment, the tool noc-generator allows to choose one of five processor types if no real-time capabilities for the routing shall be used or are required. These five processors type are ARM920T, ARM7TDMI, ARM926EJ-S, ARM Cortex A9 and ARM Cortex R4 [3]. As a consequence of these different types of processors, it is not only possible to build homogeneous architectures and systems, respectively, but also heterogeneous architectures.

By using a the tool noc-generator, a wide range of settings, concerning to the architecture of a node and the whole system, can be configured. Such settings are the overall number 
of nodes, which is limited to 64 at the moment, or if routing nodes shall be used. If computation and routing nodes shall be used, the tool allows the configuration how the notification of a computation node, if new data for even that node are available, takes place. Possible settings therefore are polling or interrupt. Also the type of routing core, which is part of the routing node, can be configured as well as frequency/mips rates of computation and routing cores.

\subsection{Routing / Communication}

Routing in our NoC design means that packets are forwarded from one core to an adjacent core on the path to the packet's destination, what corresponds to an implementation of the store and forward algorithm [12].

As already mentioned, the base for the communication between nodes and for realizing the different topology schemes are single shared memories, where specific computation and routing nodes are connected to (cf. Figure 1). These shared memories are used to hold the data/packets that have to be send from one computation node to another or that are received by a computation node. For the torus-2D topology with routing nodes, four routing nodes and cores, respectively, share such a memory (up, down, left and right).

Bounded packet sizes make memory management, flowcontrol, etc. much easier. Because we are geared towards routing around faulty or overloaded cores, we need to route messages dynamically. Thus, we prefix each message with an eight byte message header. Each hop in the network examines the message header to make routing decisions.

As the minimum, a single packet may be in flight between connected nodes at a time. To gauge the performance of this extreme point, we format the shared memory that only one packet can be placed into it at a time per attached core (single buffer). There needs to be a single buffer per direction (send and receive). Otherwise, a message sent from computation node to the other would overwrite a message concurrently sent in the opposite direction. A flag in each of the two buffers indicates whether the buffer is currently in use. The sender sets this occupied-flag once the message is completely placed into the buffer. The receiver resets the flag once the message is copied away into local memory.

A single buffer per hop and direction has one major disadvantage: The sender needs to wait until the receiver has copied away the message before it can send the next message. A slow receiver can therefore stall the sender. This problem can be solved by spending more resources (buffers) per hop and direction. To be more precise, we can format the shared memory as linked lists of messages. The user has the choice between single buffer or linked list communication.

The receiver of a message can either poll for the arrival of messages or can be notified by an interrupt that a new message has arrived. Which mechanism shall be used can be configured in the routing settings.

To implement the store and forward routing efficiently, each computation node or routing node (when they are enabled) maintains a routing table that encodes the topology of the network. To perform routing decisions, either the computation cores or the routing cores need two operations: is-responsible-for-message and is-attached-core. We need the is-responsible-for-message to be able to elect a computation core (or routing core) if multiple computation nodes (or routing nodes), attached to a given shared memory, have a link to the destination core. The operation is-attached-core is needed, that a core knows, that the destination core is one of its attached cores (and there is no need to forward the message elsewhere).

If at run-time a core or network connection dies, becomes hot, or is overloaded, a neighboring computation core or routing core can notice this and inform the others via a special broadcast message. The computation cores (or routing cores) can then patch their routing tables.

The implementation of both is-responsible-for-message and is-attached-core operations devolve into a table look-up in a pre-computed table so that the costs are negligible.

Depending on the interconnection scheme/topology and the communication/routing settings (polling or interrupt, etc.), the required communication and routing mechanism for data packets between nodes and cores, respectively, is generated in software as a library. This library has a transparent interface (API) to the programmer. As a result, the NoC software developer does not need much knowledge about the hardware.

To use our NoC design in association with our API, the programmer needs to send explicit messages. Our API is not an end-user API such as MPI or $\mathrm{MCAPI}^{1}$ but rather designed to build other APIs or hardware components on top of it. The API consists of send and receive commands (in both, blocking and non-blocking variants) to send messages up-to the packet size. The message size (MTU-size, Maximum Transmission Unit) is configurable, what results in flexibility for the user. To make the system a little more flexible, each message has an associated tag so that types of messages can be differentiated. Each node keeps a set of lists (one per tag) in its private memory. Higher level layers can then build message fragmentation/reassembly, quality of service guarantees, and high/low priority messages on top of this message tagging scheme.

\section{APPLICATIONS / USE CASES}

When a NoC design is created, the design is typically optimized for some software application area. This software application area has a range from applications that perform almost no computation but mainly communicate to applications that only compute and perform little communication. We defined a set of four software applications and use cases, respectively, that cover the extremes and single parts of these range. All applications are written in $\mathrm{C}$ and are able to scale to any number of nodes supported by the framework. As a consequence, a designer can interpolate between these use cases to get the best answers for the application profile at hand.

\subsection{Bandwidth}

The bandwidth software application and use case, respectively, simply sends a MByte of data between the nodes and then waits for a single acknowledgement message.

\subsection{Stencil kernel}

The compute-bound Stencil kernel computes the average of all the direct neighbours of each point in a matrix and writes the result into a second matrix. Each core maintains a partition of the matrices. At the end of each iteration, the boundary rows are exchanged with the adjacent cores. We

\footnotetext{
${ }^{1}$ http://www.multicore-association .org/workgroup/ mcapi.php
} 
use a $512 \cdot 512$ matrix of floats. As a result, $512 \cdot 4=2048$ bytes are transferred after each iteration and in each direction. There is a computation complexity of $\mathrm{O}\left(M^{2}\right)$ with a communication complexity of $\mathrm{O}(M)$. Communication happens rarely and in periodic bursts compared to the iteration's computation time.

\subsection{QR-codes}

QR-codes are 2D bar codes that encode a simple bit-string (a black square corresponds to 1 , a white square to 0 ) and are often printed and posted where a smartphone can take a picture of them. Because the phone's camera may be rotated with respect to the $\mathrm{QR}$-code and the QR-code may not be centered, the QR-code detection of this application rotates the picture to align the embedded QR-code and scans each rotated picture to find the QR-code's position in the picture using the corner's encoding pattern.

Computation core 0 repeatedly rotates the image by some angle (both, clockwise and anti-clockwise) and sends the results in a round-robin fashion to all other node cores in the design. These node cores scan the received and rotated image to detect a QR-code. For the application we use a $256 \cdot 256$ pixel RGB image (192 KByte) with an embedded 64.64 pixel rotated and translated QR-code. To send and receive this image the application needs to fragment the image into packets and to reassemble them. The communication pattern is thus a series of burst communications (3072 messages when using a 64 byte packet size) from core 0 to the core 1 , a small wait (while it rotates the image), then a burst of packets to core 2, and so on. Bandwidth is important for this use case.

\subsection{Packet Rewriter}

Computation core 0 creates (artificial) Ethernet packets and sends them to the other computation cores for rewriting in a round-robin fashion. Once a packet has been rewritten, it is returned to computation core 0 (which could conceptually forward it). Because an Ethernet packet can be far larger than a packet of our NoC, packet fragmentation and reassembly are needed for this application as well. We solve this problem by prefixing each packet, which is send over the NoC channels, with an extra offset field. Only after all small packets of an IP packet have arrived, the receiver rewrites the reassembled packet and sends it back to core 0 , in fragments again.

Thus, the communication pattern in this application is a repeated sequence of a set of $\left(\frac{1500}{\text { packet size }}\right)$ packets sent in a small burst from computation core 0 to each of the other computation cores followed by an almost simultaneous burst from the other computation cores back to computation core 0 . This application is mostly bandwidth-bound.

\section{RESULTS}

We distinguish our results into flexibility and simulation speed of the framework FREACSIM. Some fixed settings for the measurements and some varying settings to demonstrate the flexibility and the simulation speed are used. The focus of the flexibility measurements is on the software-based routing, not on the possibility to simulate different hardware designs and topologies. We chose only one varying parameter for the software-based routing. Considering more parameters and different hardware architectures would result in a design space exploration, what is not the goal of this paper.
The fixed settings for our measurements are elucidated in the following.

The hardware architecture used for the measurements is the $4 \times 4$ torus-2D architecture shown in Figure 1 . This is one of the most complex Network on Chip architectures the framework can generate. As a consequence, our experimental setup consists of 16 computing nodes and 16 routing nodes. The size of each shared memory is set to 256 kilobyte (KB). Because the torus-2D topology is used, a single shared memory is connected to four routing nodes for realizing the network topology. The computing nodes use the architecture, introduced in Figure 3 and section 5.1. The routing nodes use the architecture that does not support real-time ability. The type of routing core used for the routing nodes is set to ARM Cortex-R4. Our routing library without realtime ability runs on the routing cores, the computation cores use the routing library with real-time ability (eCos enabled). eCos uses the multilevel queue scheduler. The frequency of a computation core contains the value $800 \mathrm{MHz}$ and the frequency of a routing core is set to $500 \mathrm{MHz}$. For informing the computation nodes that new messages have arrived, the routing nodes use the polling strategy provided by the routing library and linked list messages for transfering data.

For each measurement, the applications elucidated in section 6 are running on the $4 \times 4$ torus-2D architecture. Each application utilizes our software base routing library and is, as a consequence, real-time capable.

Both parts of the measurements, simulation speed and flexibility of the framework, are illustrated in the following sections.

\subsection{Simulation Speed}

To show the simulation speed, and the possibility the software developer has to speed up simulation performance, we measured the simulated times of the four use cases against the wall clock times required for the simulation. Simulated time describes the overall time a use case ran on the $4 \times 4$ NoC-architecture. Wall clock time is the overall time taken by the simulation process on the host machine from start to end. We varied the parameter time slice, elucidated in section 3 , of the simulation from 1 microsecond over $5 \mathrm{mi}-$ croseconds to 25 microseconds. A packet size of 64 Bytes was used for the routing. The host machine, where the measurements were performed, was a 64 bit core i7 quad core [2] and the host operating system was fedora version 21 .

Figure 4(a) shows the simulated times of the four use cases bandwidth, stencil, QR and packet rewriter for the different time slices. Figure 4(b) shows the corresponding wall clock times. As can be seen from Figure 4(a), the simulated times vary in a small range for different time slices, although they should be the same. The reason for that circumstance are simulation artifacts, e.g. where a routing core spends time in a polling loop while waiting for the quantum to finish (see section 3$)$.

As can be seen from Figure 4(b), the larger the time slice, the shorter the wall clock time. This is the case because the simulator does not need as many context switches for large time slices as for short time slices. Setting the time slice very low delivers the most precise results, because each component simulates just a view instructions in turn. One the other hand, the wall clock time of the simulation, when setting the time slice very low, is the highest in comparison to the other time slices. 


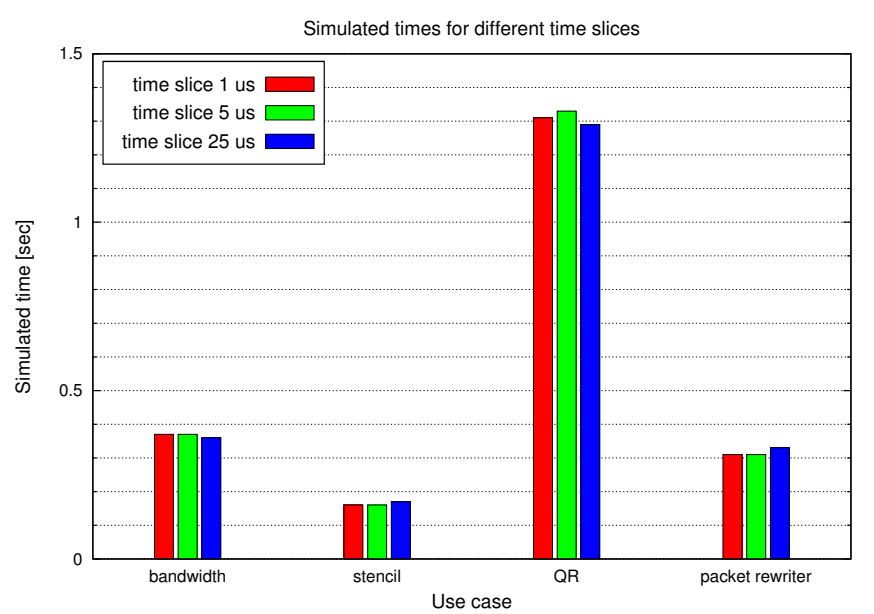

(a) Simulated times

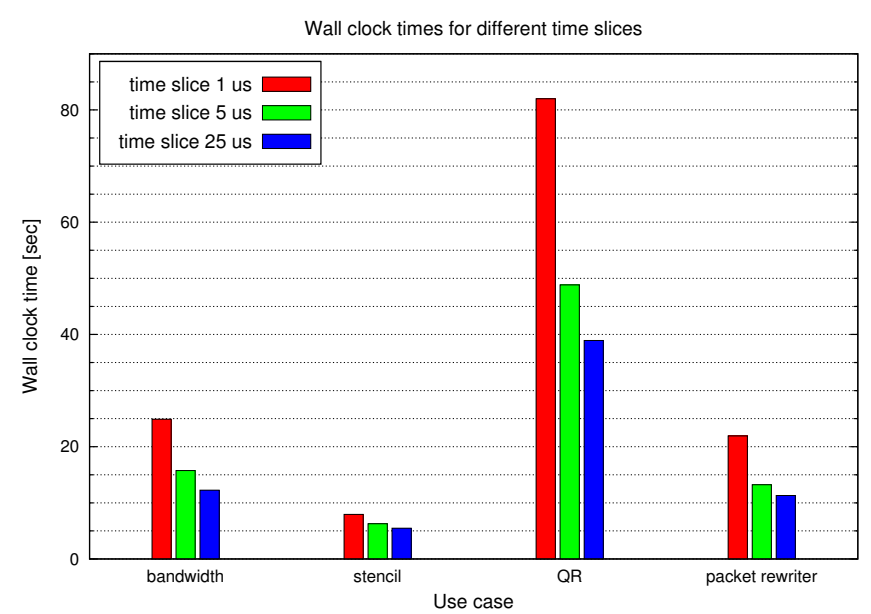

(b) Wall clock times

Figure 4: Simulated times and wall clock times of the four use cases for time slices 1us, 5us and 25 us.

For clarifying that circumstance, Figure 5 shows the average wall clock times per simulated second of the different use cases.

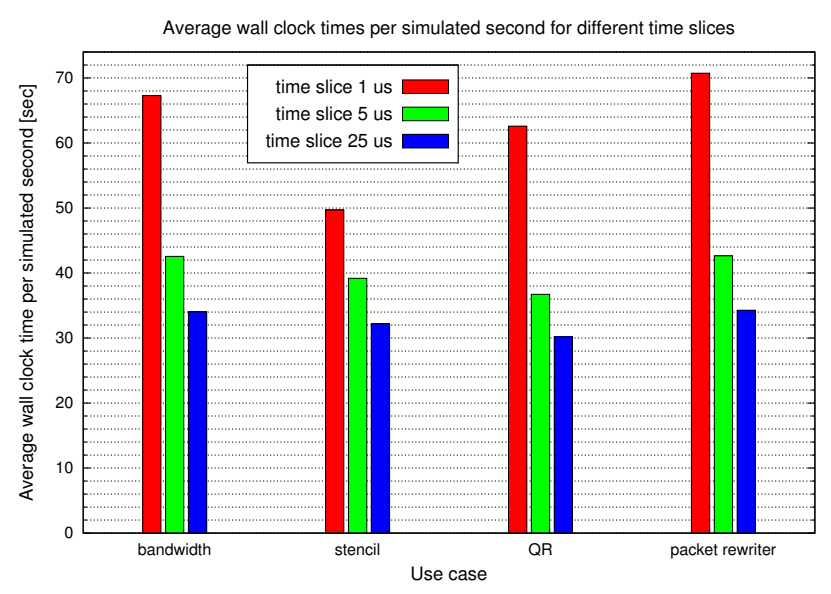

Figure 5: Average wall clock times per simulated second.

The results in Figure 5 show, that one simulated second never requires more than a wall clock time of 71 seconds, independent of the use case. One simulated second includes the simulation of 16 computation nodes as well as 16 routing nodes and the required peripherals. That corresponds to a high simulation performance and speed, respectively. As opposed to this, we measured a wall clock time of 639 seconds per simulated second for the gem 5 environment, if just a single processor of profile ARMv7-a, e.g. Cortex A9 [3], is simulated and the level of accuracy is set to low. If the level of accuracy is set to high, the wall clock time per simulated second increases to 12771 seconds (about 3.5 hours).

By using the parameter time slice, the software developer can select between simulation accuracy and simulation speed. That enables various possibilities to the software developer, concerning to the trade off of simulation accuracy and speed. If functionality of real-time capable software shall be tested, the time slice can be set to a large value, because functionality of software is not affected by the time slice.

\subsection{Flexibility}

To demonstrate the flexibility of our routing library, and the information a software developer can obtain from those flexibility, we chose one particular parameter that varies for every measurement. This parameter is the packet size. The packet size can be easily configured by setting one parameter before the build of the routing library, nothing else has to be changed. The packet size sweeps from 32 to 512 Byte and the time slice for the measurements was set to 1 microsecond. Figure 6 shows the simulated times for varying packet sizes and for the different use cases.

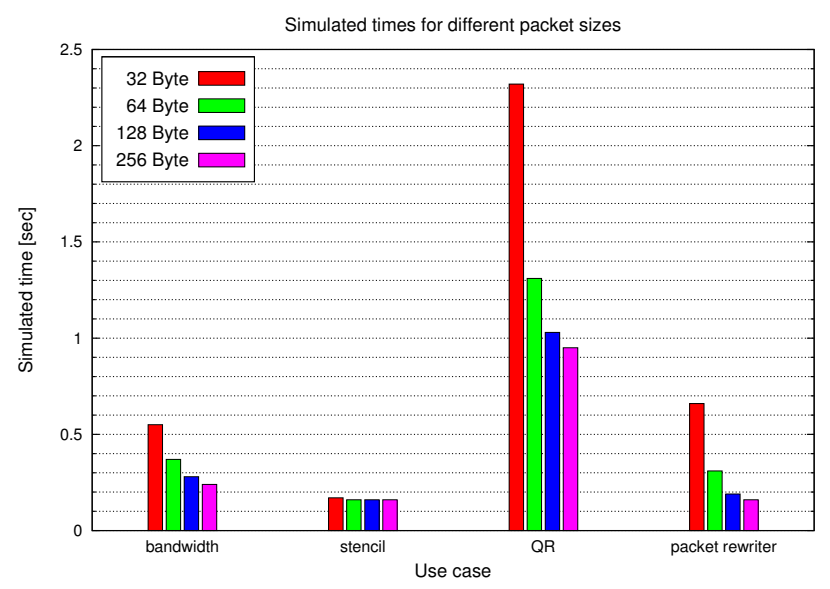

Figure 6: Simulated times for different packet sizes.

As expected, the measurements emphasize, that changing the packet size can have a decisive influence on the simulated time and, as a consequence, the run time of the use cases. The results show, that the larger the packet size, the faster the application of the respective use case. The larger the packet size, the more data can be transfered in one com- 
munication step. This is the reason why the simulated time wanes the larger the packet size.

\section{CONCLUSION}

This paper presents the highly configurable Framework for Real-time capable Embedded system and ArChitecture SIMulation (FREACSIM). To the best of our knowledge, there is no other comparable fully integrated system-simulation environment, that covers the full stack of (real-time) applications, software-based routing as well as NoC specific hardware and architecture aspects for embedded systems.

A range of embedded processors where our configurable software-based routing can be cross compiled or adapted and changed, respectively, is supported. As a result, we are able to build heterogeneous as well as homogeneous Network on Chip architectures and systems, where real-time capable and distributed software can be developed for and tested on. As a consequence, different NoC-architectures and topologies can be evaluated with the framework FREACSIM.

We allow the implementation of real-time capable and distributed applications by the usage of our routing library and the corresponding API. Software-based routing means, that special nodes implement the routing functionality in software and not in hardware, what is a good solution, if flexibility and independence shall be given. A software developer can change and adapt all parameters provided by the routing library to his own needs. This enables flexibility to the software developer and shows the influence on his software. Thus, the software developer can get a feeling how well his real-time capable software works on the respective NoC-architecture or what kind of changes have to be made.

One main advantage of our framework is the simulation speed, as illustrated in section 7 . From a point of view of a software developer, simulations of large embedded systems, like NoCs, have to be fast. Unfortunately, fast and precise simulation are in a mutual tension relationship. For the simulation of large embedded systems, its always the question, what kind of accuracy should be used. Complete cycle accurate simulations are not a good solution for such large systems, because the wall clock time of the simulation is always much greater than the simulated time. Software development does not need precise modeled hardware for testing software functionality in many cases. We think that instruction accurate simulation is the best solution for simulating distributed software functionality for such large embedded systems, even if the modelling of the hardware is not as precise as on other accuracy levels.

A further benefit of the framework is the possibility to perform design space explorations over a wide range of already available parameters. Such parameters are for example the topology of the NoC or the frequencies of computation and routing cores. Furthermore, our routing library supports a range of possible parameters, like the packet size (see Figure $4(\mathrm{a})$ ) or the organization of the shared memories (single buffer or linked list communication). This enables flexibility and allows testing software with various configurations.

Because all virtualized hardware components exist as real components, it is possible to build a real design out of the hardware components FREACSIM provides. Even our configurable software-based routing can be used on real hardware.

\section{FUTURE WORK}

In a next step, we have to validate the results of the simulator concerning to non functional properties. The instruction accurate simulation technology from OVP cannot make a clear statement about non functional properties like the execution time.

Single parts of the simulation, were precise results are necessary, could be performed on a more detailed accuracy level than FREACSIM supports at the moment. An example would be the cycle accurate level. If only single parts are of interest, not the whole simulation has to be cycle accurate for that purpose. Parts of interest can be particular memory accesses or communication traffic. In order to realize the simulation of single parts, it is necessary to switch between the accuracy levels (instruction accurate to cycle accurate and back to instruction accurate in that example).

In future work we want to tether a second simulation environment to our framework, that enables partial simulation on a more detailed level than the instruction accurate one. As a consequence of that partial simulation, the simulation speed is still high, but the results are more precise. One possible solution for that purpose would be SystemC, but there are also other solutions and simulation environments, respectively. Our XML interface between the tools noc-generator and xml-to-sim-model (refer to Figure 2 and section 5 ) is therefore a perfect point to start from.

Also, we will think about the integration of higher-level parallel programming models like OpenMP ${ }^{2}$ and $\mathrm{MPI}^{3}$.

\section{REFERENCES}

[1] ecos on arm integrator compact platform. www.ovpworld.org/operating-systems-support-ecos, 2015. Last visit on 04.02.2015.

[2] Intel core i7-4702mq processor website. http://ark.intel.com/de/products/75119/Intel-Corei7-4702MQ-Processor-6M-Cache-up-to-3_20-GHz, 2015. Last visit on 04.02.2015.

[3] Official arm website for processors. http://www.arm.com/products/processors/index.php, 2015. Last visit on 02.02.2015.

[4] Official ecos website. http://ecos.sourceware.org/, 2015. Last visit on 04.02.2015.

[5] Official gems website. http://research.cs.wisc.edu/gems/, 2015. Last visit on 02.02.2015.

[6] Official systemc website. http://www.systemc.org, 2015. Last visit on 02.02.2015.

[7] N. Agarwal, T. Krishna, L.-S. Peh, and N. Jha. Garnet: A detailed on-chip network model inside a full-system simulator. In Performance Analysis of Systems and Software, 2009. ISPASS 2009. IEEE International Symposium on, pages 33-42, April 2009.

[8] F. Bellard. Qemu, a fast and portable dynamic translator. In USENIX Annual Technical Conference, FREENIX Track, pages 41-46, 2005.

[9] N. Binkert, B. Beckmann, G. Black, S. K. Reinhardt, A. Saidi, A. Basu, J. Hestness, D. R. Hower, T. Krishna, S. Sardashti, R. Sen, K. Sewell, M. Shoaib, N. Vaish, M. D. Hill, and D. A. Wood. The

${ }^{2}$ http://openmp.org/wp/

${ }^{3}$ http://www.mcs.anl.gov/research/projects/mpi/ 
gem5 simulator. SIGARCH Comput. Archit. News, 39(2):1-7, Aug. 2011.

[10] N. L. Binkert, R. G. Dreslinski, L. R. Hsu, K. T. Lim, A. G. Saidi, and S. K. Reinhardt. The $\mathrm{m} 5$ simulator: Modeling networked systems. IEEE Micro, 26:52-60, 2006.

[11] Boson. Boson NetSim 10 User Manual. Boson Software, LLC, 25 Century Blvd., Ste. 500, Nashville. Last access date: 02.10.2014.

[12] R. Cypher, F. Meyer auf der Heide, C. Scheideler, and B. Vöcking. Universal algorithms for store-and-forward and wormhole routing. In Proceedings of the Twenty-eighth Annual ACM Symposium on Theory of Computing, STOC '96, pages 356-365, New York, NY, USA, 1996. ACM.

[13] Imperas Software Limited. OVP Guide to Using Processor Models. Imperas Buildings, North Weston, Thame, Oxfordshire, OX9 2HA, UK, May 2014. Version 0.5, docs@imperas.com.

[14] Imperas Software Limited. OVPsim and Imperas CpuManager User Guide. Imperas Buildings, North Weston, Thame, Oxfordshire, OX9 2HA, UK, August 2014. Version 2.3.6, docs@imperas.com.

[15] N. Jiang, D. Becker, G. Michelogiannakis, J. Balfour, B. Towles, D. Shaw, J. Kim, and W. Dally. A detailed and flexible cycle-accurate network-on-chip simulator. In Performance Analysis of Systems and Software (ISPASS), 2013 IEEE International Symposium on, pages 86-96, April 2013.

[16] T. Karadeniz, L. Mhamdi, K. Goossens, and J. Garcia-Luna-Aceves. Hardware design and implementation of a network-on-chip based load balancing switch fabric. In Reconfigurable Computing and FPGAs (ReConFig), 2012 International Conference on, pages 1-7, Dec 2012.

[17] J. Madsen, S. Mahadevan, K. Virk, and M. Gonzalez. Network-on-chip modeling for system-level multiprocessor simulation. In Real-Time Systems Symposium, 2003. RTSS 2003. 24th IEEE, pages 265-274, Dec 2003.

[18] A. J. Massa. Embedded Software Development with eCos. Prentice Hall Professional Technical Reference, December 2002.

[19] J. Miller, H. Kasture, G. Kurian, C. Gruenwald, N. Beckmann, C. Celio, J. Eastep, and A. Agarwal. Graphite: A distributed parallel simulator for multicores. In High Performance Computer Architecture (HPCA), 2010 IEEE 16th International Symposium on, pages 1-12, Jan 2010.

[20] L. Ni and P. McKinley. A survey of wormhole routing techniques in direct networks. Computer, 26(2):62-76, Feb 1993.

[21] D. Schoenwetter, V. Sieh, and D. Fey. Porting an engine control application to a virtual environment by using an open source real time operating system. In DESIGN\&ELEKTRONIK, editor, Embedded World Conference Proceedings, 2013, Nuremberg, feb. 2013. WEKA FACHMEDIEN GmbH, Haar.

[22] SoClib. Official soclib developer website. http://www.soclib.fr/trac/dev, 2015. Last visit on 01.02 .2015 .

[23] V. M. Weaver and S. A. McKee. Are cycle accurate simulations a waste of time? In Proc. 7th Workshop on Duplicating, Deconstructing, and Debunking, June 2008. 